\title{
Anemia in Pregnancy \& Parenteral Iron Therapy
}

\section{Raúl H Morales-Borges ${ }^{1,2,3^{*}}$}

${ }^{1}$ Ashford Institute of Hematology \& Oncology, San Juan, PR, USA

${ }^{2}$ American Red Cross, Puerto Rico Region, USA

${ }^{3}$ Ashford Presbyterian Community Hospital, San Juan, PR, USA

\begin{abstract}
Anemia is common in pregnancy and iron deficiency is the most common cause. Oral iron is the standard therapy, but there is a problem of adherence due the gastrointestinal side effects. Intravenous iron therapy has more advantages, therefore, intravenous iron therapy is the best treatment for pregnant women during the third trimester. We reviewed the statistics of deliveries of the fiscal year 2012, the cases of anemia in pregnancy as in patients provided by Record Room from the calendar year 2012 and the patient cases that used sodium ferric gluconate complex in sucrose injection (FERRLECIT) from the Obstetrics/Gynecology Ward from the fiscal year 2012 provided by the Department of Pharmacy of the Ashford Presbyterian Community Hospital (APCH). A review of the records of pregnant women receiving iron dextran injection (INFeD) intravenously in our ambulatory infusion center was also performed. Our data confirmed that either presentations elevated the hemoglobin and they were well tolerable. Iron sucrose is the preferred and it has demonstrated a high success rate.

I recommend considering this alternative earlier at the third trimester and establishing guidelines using parenteral iron and erythropoietin in combination in refractory cases.
\end{abstract}

Keywords: Anemia; Pregnancy; Parenteral; Iron

\section{Introduction}

Anemia is a frequent condition during pregnancy, particularly among women from developing countries who have insufficient iron intake to meet increased iron needs of both the mother and the fetus. Traditionally, gestational anemia has been prevented with the provision of daily iron supplements throughout pregnancy, but adherence to this regimen due to side effects, interrupted supply of the supplements, and concerns about safety among women with an adequate iron intake, have limited the use of this intervention. Intermittent (i.e. one, two or three times a week on non-consecutive days) supplementation with iron alone or in combination with folic acid or other vitamins and minerals has recently been proposed as an alternative to daily supplementation [1]. Intermittent iron and folic acid regimens produce similar maternal and infant outcomes at birth as daily supplementation but are associated with fewer side effects. Women receiving daily supplements had increased risk of developing high levels of hemoglobin $(\mathrm{Hb})$ in mid and late pregnancy but were less likely to present mild anemia near term. Although the evidence is limited.

Large doses of iron are most often prescribed and are associated with side effects and with increased oxidative damage. Alternatively, delayed-release preparations and intermittent oral iron supplementation lead to better overall compliance and alleviate side effects. Daily iron intervention provides more protection against a decline in the storage iron pool in pregnant women than does an intermittent schedule, but the latter is generally associated with fewer side effects, better compliance, and possibly a reduction in risk of oxidative damage. An improved cost-benefit ratio associated with a lower-dose oral iron supplement may prove to be quite positive in the future. Currently, no single approach may be universally acceptable, although a moderate iron dosage protocol will likely provide the most benefit to those who require supplemental iron [2].

Parenteral iron seems to be an alternative and it is well known since 1960 [3]. It is often required in many pregnant women. It is preferred whenever they presented with failure to oral iron or increased demands despite oral iron therapy. There are two presentations: iron dextran and iron sorbitol citric acid [4]. Anaphylactic reaction is the principal problem with iron dextran, but iron sucrose is safe and effective without requirement of a test dose [5-7]. They are given is most of the ambulatory infusion clinics or centers with minimal facilities. Therefore, this review was aimed to comparing the efficacy and safety of parenteral iron therapy of our practice with the current worldwide practice in this area.

\section{Methods}

I reviewed the statistics of deliveries (including cesarean section) of the fiscal year 2012 (July 2012 to June 2013) provided by Labor \& Delivery Room of the Ashford Presbyterian Community Hospital (APCH), the cases of anemia in pregnancy (ICD-9 codes 648.23, 648.21) as in patients provided by Record Room of APCH from the calendar year 2012 (January 1 to December 31 of 2012), and the in patient cases that used sodium ferric gluconate complex in sucrose injection (FERRLECIT) from the Obstetrics/Gynecology Ward of the same hospital from the fiscal year 2012 provided by the Department of Pharmacy of APCH. The hospital is a community based non-profit health care facility of 200 beds with ambulatory and in patient services located at the Condado Area of San Juan, Puerto Rico. Their expertise are obstetrics (in particularly, high risk pregnant women), orthopedics, internal medicine, and ambulatory surgery.

The records from my practice were reviewed too regarding the pregnant women referred to me who required parenteral iron therapy. We paid attention to the $\mathrm{Hb}$ and the Hematocrit (Hct). Serum iron and

*Corresponding author: Raúl H. Morales-Borges, MD, Ashford Institute of Hematology \& Oncology, 29 Washington Street, Suite \# 107, San Juan, PR, 00907-1509, USA, Tel: (787) 722-0412; Fax: (787) 723-0554; E-mail: rmoralesborges@yahoo.com

Received August 31, 2013; Accepted September 20, 2013; Published September 24, 2013

Citation: Morales-Borges RH (2013) Anemia in Pregnancy \& Parenteral Iron Therapy. J Blood Disorders Transf 4: 171. doi: 10.4172/2155-9864.1000171

Copyright: (c) 2013 Morales-Borges RH. This is an open-access article distributed under the terms of the Creative Commons Attribution License, which permits unrestricted use, distribution, and reproduction in any medium, provided the original author and source are credited. 
ferritin levels were not recorded in this review in view that most of the cases did not have those tests.

I have 18 years as solo practice of hematology/oncology near by the $\mathrm{APCH}$ and I am evaluating and treating hematological complications in obstetrics, pregnancy, and gynecology for the last 15 years. We used iron dextran injection (INFeD) intravenously in our ambulatory infusion center.

The author critically reviewed the evidence, and the information was augmented by a computerized PubMed search of the English and Spanish language literature published across the years to the present. The information was correlated with the above data mentioned.

\section{Results}

There were a total of 3,643 deliveries in the fiscal year 2012 at APCH with values between 230-331 deliveries per month for an average of 280 deliveries per month.

There were 341 admissions in the calendar year 2012 with an average of 28 admissions per month with the ICD-9 codes 648.23 and 648.21 as per the data from the record room of the hospital. The majorities were at third trimester (76\%) and only $24 \%$ were at second trimester. The age of the patients is from 14 to 43 with an average age of 31 . That means that the $10 \%$ of the pregnant women have anemia in our institution.

Regarding parenteral iron therapy as in patient, there were a total of 67 cases that FERRLECIT was indicated of which 55 were pregnant for $82.09 \%$. Their age is from 15 to 38 with an average age of 27.25 . They have an average of 4.58 pregnant women per month that receive parenteral iron, so, $46 \%$ of the pregnant women with anemia in the hospital receive intravenous iron therapy. The utilized dose was $125 \mathrm{mg}$ daily for $94.55 \%$ and $62.5 \mathrm{mg}$ for $5.45 \%$. The most common adverse reactions reported were pruritus, chest pain, abdominal pain, headaches, dizziness, and hypotension but they were mild and manageable.

According the data from my practice, we have 240 office visits of which 20 referrals $(8.3 \%)$ are pregnant women with anemia per month and we are treating 15 with parenteral iron for $75 \%$. Their age is from 19 to 38 and the average age of 27 . All the cases (100\%) are at third trimester and $89 \%$ were at $32-36$ weeks of gestation. The $\mathrm{Hb}$ pre infusion was from $7.1 \mathrm{gm} / \mathrm{dl}$ to $10.5 \mathrm{gm} / \mathrm{dl}$ with an average of 8.8 $\mathrm{gm} / \mathrm{dl}$. The medication that we use is INFeD and the dose is $100 \mathrm{mg}$ intravenously in $0.9 \%$ normal saline solution to be infused in one hour twice or three times per week for a total of 6 to 10 infusions in 3 to 4 weeks. The $\mathrm{Hb}$ post infusion was from $9.8 \mathrm{gm} / \mathrm{dl}$ to $12.1 \mathrm{gm} /$ dl. We noticed an increment in $\mathrm{Hb}$ of 1 to $2 \mathrm{gm} / \mathrm{dl}$. The most common adverse reactions were dermatologic such as rash, urticaria, hives, and pruritus. Once we identify the adverse reactions, we administer diphenhydramine (Benadryl) immediately and afterwards. There was only one case of syncope and another case of chest tightness.

For purpose of the review, we choose 10 cases randomly by medical record number of patients seen on year 2012 (Table 1).

\section{Discussion}

Approximately $51 \%$ of pregnant women are anemic. This includes a prevalence of $56 \%$ in developing countries and $18 \%$ in developed countries. Among these, $43 \%$ of women from developing countries and $12 \%$ of women from developed countries were already anemic, preconception. The WHO has estimated that considering all forms of anemia, from 16,800 to 28,000 women of reproductive age die annually from anemia, with the greatest risk in younger women. Of all anemia's diagnosed during pregnancy, $75 \%$ are due to iron deficiency [8]. The incidence of anemia in pregnant women in India is 54.6\% in urban and $59 \%$ in rural areas, and South Asian countries have the highest among the other countries in the world [9]. In our hospital, $10 \%$ of our pregnant women have anemia and in my practice, $8.3 \%$. There is no study reported in the medical literature indicating the incidence and prevalence in Puerto Rico.

By age, women within ages 20 to 49 have a prevalence of iron deficiency anemia of $11 \%$ and non-pregnant of the same range of age with $5 \%$. They have anemia most commonly at the third trimester with a prevalence of $37 \%$ (8). We concurred on comparison with our hospital and office data. The average age was 27 with ages between 21 and 32 as per Table 1 and the gestational age of 21 to 38 weeks. At the hospital the average age was 31 and they had the youngest cases of 14 .

The grade of anemia registered from us is very similar in comparison with the ones from the medical literature $(1-4,10-13)$. The normal $\mathrm{Hb}$ value during pregnancy on 5th percentile by 28 weeks is $10.7 \mathrm{gm} / \mathrm{dl}$ and by 36 weeks is $11.4 \mathrm{gm} / \mathrm{dl}[8]$.

Regarding parenteral (intravenous) iron therapy, our data confirmed that either presentations elevated the $\mathrm{Hb}$ or they were well tolerable. Perewusnyk et al., found that accumulation of iron sucrose in parenchyma of organs is low compared with iron dextrans or iron gluconate, while incorporation into bone marrow for eryhtropoiesis is considerably faster, so, that is the reason why parenteral iron sucrose is used primarily treating the anemia efficiently and safety reducing the rate of blood transfusion to below $1 \%$ of patients per year $[9,10]$. According to Shafi et al., the difference in $\mathrm{Hb}$ values from baseline was $1.72 \mathrm{gm} / \mathrm{dl}$ at 2 weeks, $2.18 \mathrm{gm} / \mathrm{dl}$ at 4 weeks, and $2.89 \mathrm{gm} / \mathrm{dl}$ at 6 weeks [9]. We obtained a difference of $2 \mathrm{gm} / \mathrm{dl}$ at 3-4 weeks with a total of 8-10 infusions. We are very similar to the results obtained by $\mathrm{T}$. Mays and Mays with an average $\mathrm{Hb}$ response of $1.9 \mathrm{gm} / \mathrm{dl}$ [11] Interestingly, Carretti and Paticchio from Italy found that the response to intravenous iron therapy in pregnancy anemia is related to $\mathrm{Hb}$ level and gestational age at the onset of treatment and probably depends on the erythropoietin response to anemia [12]. This is the reason why Perewusnyk et al. recommended that in cases of severe anemia ( $\mathrm{Hb}$ below $9 \mathrm{~g} / \mathrm{dl}$ ) or non-response to parenteral iron after 2 weeks, recombinant erythropoietin is considered in combination [10].

Parenteral iron is available for patients with mal-absorption syndrome and severely anemic patients who refuse oral iron supplementation. Iron dextran can be given intramuscularly or intravenously. Anaphylactic reactions have been reported in $1 \%$ of patients receiving iron dextran, but in our experience we did not have any case and side effects were rare and manageable. Intravenous iron therapy has been shown to replenish iron stores and raises hemoglobin levels faster than oral iron, but no long-term benefits have been observed. Erythropoietin with or without iron supplementation can safely be used to treat severe iron deficiency anemia in pregnancy. When compared with iron supplementation alone, the addition of erythropoietin to iron supplement has been shown to increase reticulocyte count and hematocrit faster, and decrease the time required to reach target hemoglobin levels [13].

\section{Conclusion}

Iron sucrose as well as iron dextran are an effective treatment for anemia in pregnancy. They are safe, efficacious, fast, and cost effective as an alternative in the management of anemia in pregnant women at third trimester. As per the majority of studies, iron sucrose is the preferred and they demonstrated a high success rate, better compliance, 
Citation: Morales-Borges RH (2013) Anemia in Pregnancy \& Parenteral Iron Therapy. J Blood Disorders Transf 4: 171. doi: 10.4172/21559864.1000171

Page 3 of 3

\begin{tabular}{|c|c|c|c|c|c|c|c|}
\hline Case \# & Age (years old) & Gest. Age (weeks) & \# of Tx & Duration of Tx (weeks) & $\mathrm{Hb} / \mathrm{Hct}$ Pre & Hgb/Hct Post & Diff. in $\mathrm{Hb}$ (g/dl) \\
\hline 1 & 21 & 32 & 9 & 2 & $8.2 / 24.3$ & $9.9 / 30.1$ & 1.7 \\
\hline 2 & 21 & 32 & 10 & 4 & $9.07 / 29.6$ & $11.5 / 36.7$ & 2.43 \\
\hline 3 & 32 & 33 & 6 & 3 & $9.59 / 30.9$ & $11.5 / 35.5$ & 1.91 \\
\hline 4 & 28 & 38 & 3 & 1 & $10.1 / 31.5$ & $11.9 / 34.5$ & 1.9 \\
\hline 5 & 27 & 29 & 10 & 4 & $8.1 / 26.9$ & $11.6 / 36.3$ & 3.5 \\
\hline 6 & 26 & 34 & 10 & 4 & $8.6 / 25.7$ & $11.2 / 33.9$ & 2.6 \\
\hline 7 & 28 & 34 & 12 & 4 & $9.6 / 28.7$ & $11.4 / 33$ & 1.8 \\
\hline 8 & 25 & 21 & 14 & 4 & $8.8 / 27.2$ & $9.8 / 30.9$ & 1 \\
\hline 9 & 31 & 33 & 8 & 4 & $10.5 / 30.6$ & $12.1 / 36$ & 1.6 \\
\hline 10 & 32 & 29 & 9 & 6 & $9.5 / 29.1$ & $11.8 / 35.7$ & 2.3 \\
\hline Max & 32 & 38 & 14 & 6 & $10.5 / 31.5$ & $12.1 / 36.7$ & 3.5 \\
\hline Min & 21 & 21 & 3 & 1 & $8.1 / 24.3$ & $9.8 / 30.1$ & 1 \\
\hline Average & 27 & 31.2 & 9 & 3.6 & $9.2 / 28.5$ & $11.3 / 34.3$ & 2.1 \\
\hline
\end{tabular}

$\left(^{*}\right)$ Legend of abbreviations used: \# - number, Gest.- Gestational, Tx-Treatments, Diff - Difference

Table 1: Data of pregnant women on intravenous iron from Dr. Morales-Borges Office $\left(^{*}\right)$.

and a decrease in the rate of transfusion use post partum. I recommend considering this alternative earlier at the third trimester and establishing guidelines using parenteral iron and erythropoietin in combination in refractory cases.

\section{Acknowledgements}

We thank to the staff from record room, Mrs. Diaz (nurse manager) from labor and delivery room, Nancy I. Morales-Berrios, Pharm.D (Clinical Pharmacist Specialist) from Comprehensive Pharmacy Services, and Dr. Francisco de Torres (Medical Director) for their collaboration in obtaining the data from the Ashford Presbyterian Community Hospital in San Juan, PR.

I am very grateful of Mrs. Martha Otero (Oncology/Infusion Nurse) and Mrs. Nilsa Rivera (Office Manager) from my office (Ashford Institute of Hematology \& Oncology) for the care of the patients and their assistance in collecting the data.

\section{References}

1. Peña-Rosas JP, De-Regil LM, Dowswell T, Viteri FE (2012) Intermittent ora iron supplementation during pregnancy. Cochrane Database Syst Rev 7: CD009997.

2. Beard JL (2000) Effectiveness and strategies of iron supplementation during pregnancy. Am J Clin Nutr 71: 1288S-94S.

3. hapke Fb, Stauf Jl, Ullery Jc (1960) Parenteral iron therapy in anemia of pregnancy. Am J Obstet Gynecol 79: 886-891.

4. Dhanani JV, Ganguly BP, Chauhan LN (2012) Comparison of efficacy and safety of two parenteral iron preparations in pregnant women. J Pharmacol Pharmacother 3: 314-319.
5. Van Wyck DB, Cavallo G, Spinowitz BS, Adhikarla R, Gagnon S, et al. (2000) Safety and efficacy of iron sucrose in patients sensitive to iron dextran: North American clinical trial. Am J Kidney Dis 36: 88-97.

6. Silverstein SB, Rodgers GM (2004) Parenteral iron therapy options. Am J Hematol 76: 74-78.

7. Faich G, Strobos J (1999) Sodium ferric gluconate complex in sucrose: safer intravenous iron therapy than iron dextrans. Am J Kidney Dis 33: 464-470.

8. Baker WF, Lee R (2006) Iron deficiency, folate, and vitamin B12 deficiency in pregnancy, obstetrics, and gynecology. In: Bick RL, Frenkel EP, Baker WF Sarode R (Eds.), Hematological Complications in Obstetrics, Pregnancy and Gynecology, Cambridge University Press, United Kingdom 269-309.

9. Shafi D, Purandare SV, Sathe AV (2012) Iron deficiency anemia in pregnancy: intravenous versus oral route. J Obstet Gynaecol India 62: 317-321.

0. Perewusnyk G, Huch R, Huch A, Breymann C (2002) Parenteral iron therapy in obstetrics: 8 years experience with iron-sucrose complex. Br J Nutr 88: 3-10.

11. Mays T, Mays T (1976) Intravenous iron-dextran therapy in the treatment of anemia occurring in surgical, gynecologic and obstetric patients. Surg Gynecol Obstet 143: 381-384.

12. Carretti N, Paticchio MR (1999) Intravenous iron therapy in pregnancy anemia hematological response in relation to gestational age. Gynecol Obstet Invest 47: $217-222$

13. Horowitz KM, Ingardia CJ, Borgida AF (2013) Anemia in pregnancy. Clin Lab Med 33: 281-291. 